\title{
Investigation of the Effects and Mechanisms of Mai Tong Formula on Lower Limb Macroangiopathy in a Spontaneous Diabetic Rat Model
}

\author{
Guangming Gong, Haipo Yuan, Ya Liu, and Luguang Qi \\ Endocrinology Department, Teaching Hospital of Chengdu University of Traditional Chinese Medicine, Chengdu 610075, China \\ Correspondence should be addressed to Guangming Gong; 13980506075@163.com
}

Received 20 September 2016; Accepted 19 October 2016

Academic Editor: Nikolaos Papanas

Copyright (C) 2016 Guangming Gong et al. This is an open access article distributed under the Creative Commons Attribution License, which permits unrestricted use, distribution, and reproduction in any medium, provided the original work is properly cited.

\begin{abstract}
A new Chinese herbal formula called Mai Tong Formulae (MTF) has recently been used to treat lower limb macroangiopathy in type 2 diabetes mellitus (T2DM) patients. In this study, we investigated the effect of MTF on lower limb macroangiopathy in a spontaneous diabetic rat model (GK rats). We found that MTF treatment significantly reduced serum fasting blood glucose (FBG), triglycerides (TG), total cholesterol (TC), IL6, and VEGF and increased serum insulin in this model. Histological and ultrastructural observations showed that MTF treatment significantly reduced vascular endothelial cell shedding and improved endothelium injuries. We further detect proteome alteration following MTF treatment. 25 differential proteins (DPs) abnormally expressed in GK rats were normalized by MTF treatment. These DPs significantly are enriched in biological processes and pathways that regulate muscle contraction and cGMP-PKG signaling pathway and so on. Additional protein-protein interaction (PPI) network analyses of the DPs showed that Fasn and Prkar2a are involved in the AMPK signaling pathway, and Gnas, Myhll, and Myh6 are involved in vascular smooth muscle contraction; these 5 DPs were validated by Western blotting. These results indicate that MTF treatment effectively treats lower limb macroangiopathy by regulating key proteins involved in AMPK signaling pathway and vascular smooth muscle contraction.
\end{abstract}

\section{Introduction}

The prevalence of diabetes mellitus is increasing dramatically worldwide. Reports showed that, in 2015 , over $8 \%$ of adult people worldwide suffer from type 2 diabetes mellitus (T2DM) (International Diabetes Federation, 2015) [1]. Lower limb macroangiopathy is a major complication T2DM which is caused by various pathogenic factors, including blood glucose levels, peripheral neuropathy, oxidative stress, and ischemia $[2,3]$. Lower limb macroangiopathy can develop into lower limb ulcers, which decrease quality of life and contribute to high morbidity, mortality, and healthcare costs [4]. Currently, there is no medication to cure T2DM or macroangiopathy complications. In Western medicine, some blood glucose-lowering drugs, such as rosiglitazone and glibenclamide, have been used to alleviate the symptoms of T2DM but significant side effects and drug resistance exist [5]. Therefore, the development of novel longer-lasting, targeted therapeutics is urgently needed.
In China, traditional herbal formulas have been used for centuries to treat T2DM [6, 7]. Many herbal extracts can reduce blood glucose and improve complications associated with T2DM [8-11], and, compared to Western medications, many Chinese herbs have fewer side effects [12]. However, the complexity and various actions of herbal components have limited their application worldwide.

Recently, a new herbal formula called Mai Tong Formula (MTF) has been used to treat lower limb macroangiopathy and diabetic foot ulcers (DFU) in T2DM patients and has a curative effect on hyperglycemia, atherosclerosis, nephropathy, and inflammation [13]. However, the effect of MTF treatment on lower limb macroangiopathy has not been evaluated systematically, and no studies have explored the molecular mechanism of this herbal formula. Herein, we aim to explore the effect and molecular mechanism of MTF using a spontaneous diabetic rat model (GK rats). First, we evaluated the effect of MTF by measuring several blood biochemical indicators and histological observation; then, we employed 
quantitative proteomic assays using isobaric tags for relative and absolute quantitation (iTRAQ), combined with high performance liquid chromatography-tandem mass spectrometry (HPLC-MS/MS), to detect proteome alteration from MTF treatment. Additional bioinformatics analyses were used to analyze the differential proteins (DPs) to investigate the key pathways underlying the mechanism of MTF treatment.

\section{Materials and Methods}

2.1. Animal Model and MTF Preparation. MTF is composed of dozens of herbs, including Huangqi (Radix Astragali), Sangshen (Fructus Mori), Danggui (Angelica sinensis), Danshen (Salvia miltiorrhiza Bge.), Zexie (Alisma plantagoaquatica Linn.), and Yinhuateng (Lonicera japonica Thunb.). All herbs were decocted with water, filtered, and brought to a final concentration of $1.0 \mathrm{~g} / \mathrm{mL}$.

Experimental protocols were approved by the Experimental Animal Care and Ethics Committees of the Teaching Hospital of Chengdu University of Traditional Chinese Medicine. Seven normal rats and 14 spontaneous diabetic rat models (Goto-kakizaki rats, GK rats) were purchased from SLRC Laboratory Animal Co., Ltd. (Shanghai, China). All rats were 8-week-old females with a body weight between $150 \mathrm{~g}$ and $210 \mathrm{~g}$ (certification number: SCXK (hu) 2003-0003). Seven normal rats were used as a control group and were given $2 \mathrm{~mL}$ intragastric saline vehicle $(0.9 \%)$ once a day for 12 weeks. $14 \mathrm{GK}$ rats were randomized and divided into two groups: (1) the model group $(n=7)$ was given $2 \mathrm{~mL}$ of intragastric saline vehicle $(0.9 \%)$ once a day for 12 weeks; (2) the MTF group $(n=7)$ was given $29 \mathrm{~g} / \mathrm{kg}$ MTF decoction once a day for 12 weeks. All rats were anesthetized and sacrificed under the experimental protocols described above and all efforts were made to minimize suffering.

2.2. Blood Indicators Examination. We removed the rats' tails and obtained samples to measure fasting blood glucose (FBG), blood triglycerides (TG), and blood total cholesterol (TC) levels in the 12th week of testing (AU5800, Beckman Coulter, USA). Serum insulin was measured by radioimmunoassay (HTA Co., Ltd., Beijing, China, number 2013009) using $2 \mathrm{~mL}$ inner canthus blood. Vascular endothelial growth factor (VEGF) and serum interleukin 6 (IL6) were measured by double-antibody sandwich ELISA (BOSTER Inc., Wuhan, China, number 2013006) using $2 \mathrm{~mL}$ inner canthus blood.

2.3. Pathologic Histology. For histological observation, rat femoral arteries were excised and fixed in $10 \%$ neutral formalin paraffin-embedded after dehydration; then, sections of tissue were stained with hematoxylin and eosin and images were obtained by light microscopy (Olympus, Tokyo, Japan). For ultrastructural observation, the femoral arteries were excised and fixed in 3\% glutaraldehyde $\left(4^{\circ} \mathrm{C}\right)$, dehydrated, embedded, and cut into semithin sections for optical localization; ultrathin sections were stained and visualized by electron microscopy (JEM-1010 (HC), JEOL).
2.4. Proteomic Analysis. $0.7 \mathrm{~g}$ of femoral arteries from 7 rats of each group $(0.1 \mathrm{~g}$ per rat) was collected for protein extraction; then, protein $(100 \mu \mathrm{g})$ was digested with trypsin for $12 \mathrm{~h}$ at $37^{\circ} \mathrm{C}$ (protein/enzyme $\left.=100 / 3.3\right)$. After iTRAQ $(A B$ Science) labeling, equal amounts of labeled peptides from each group were mixed and resolved into 15 fractions by high performance liquid chromatography (HPLC), followed by Q Exactive mass spectrometry (Thermo Fisher Scientific). The resulting MS/MS data were qualitatively and quantitatively analyzed by Mascot 2.3.01 with the following parameters: protein identification using the nonredundant International Protein Index rat protein database (version 3.72) and full trypsin digest with a maximum of 1 missed cleavage. Peptide tol. and MS/MS tol. were 0.05 Da. Scaffold software was used to identify the differential proteins (Dps). Proteins with $P<$ 0.05 fold change higher than 1.2 or lower than 0.833 were DPs.

2.5. Western Blotting Analysis. Cell lysates were separated by SDS-PAGE in 8\% Tris-glycine gels (Invitrogen Life Technologies, Carlsbad, CA, USA) and transferred to a nitrocellulose membrane. Blots were probed with specific antibodies [diluted with $5 \%$ bovine serum albumin (BSA) to $1: 1000$ ]. Membranes were probed with horseradish peroxidaselabeled anti-rabbit secondary antibody (diluted with 5\% BSA to 1:1000; Cell Signaling Technology, Inc., Danvers, MA, USA). Antibody binding was detected by using an enhanced chemiluminescence detection kit (Amersham International PLC, Buckinghamshire, UK).

2.6. Data Preprocessing. The data are presented as mean \pm standard deviation. Statistical comparisons among the three experimental groups were made using unpaired Student's $t$ tests. The GO and KEGG pathway enrichment analysis of DPs were performed using the Database for Annotation, Visualization, and Integrated Discovery (DAVID) [14]. The protein-protein interaction (PPI) networks were constructed using the String 10.0 database [15].

\section{Results}

3.1. Blood Indicators Examination and Pathologic Histology. From 0 to 12 weeks, compared with the control group, the rats in the model group were inactive, withered, and lusterless and had sparse fur, experienced diarrhea, and consumed more food and water. These symptoms in the MTF group were significantly alleviated.

We detected FBG, TG, and TC for all three experimental groups in the 12th week. As the results show in Figures 1(a), 1(b), and 1(c), the levels of FBG, TG, and TC in the model group were significantly higher than in the control group $(P<0.01)$, and, compared to model group, FBG, TG, and TC levels in the MTF group significantly improved $(P<0.01)$. As shown in Figure 1(d), serum insulin levels in the model group were significantly lower than in the control group $(P<0.01)$, and, compared to the model group, serum insulin levels in the MTF group were significantly increased $(P<0.01)$. As shown in Figures 1(e) and 1(f), serum VEGF and IL6 levels were significantly higher than in the control group $(P<0.01)$, 


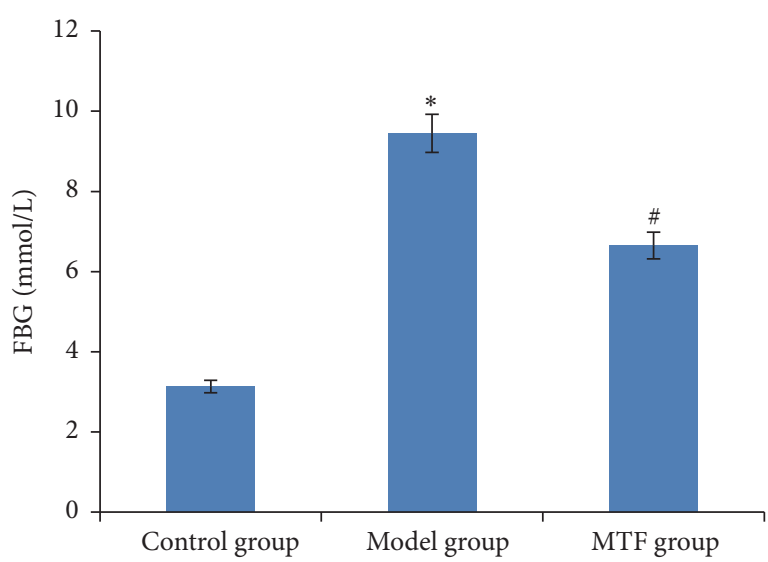

(a)

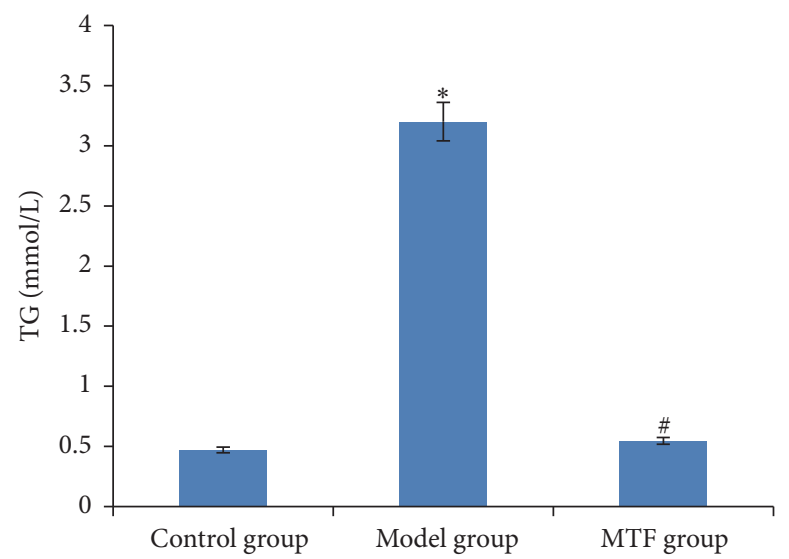

(c)

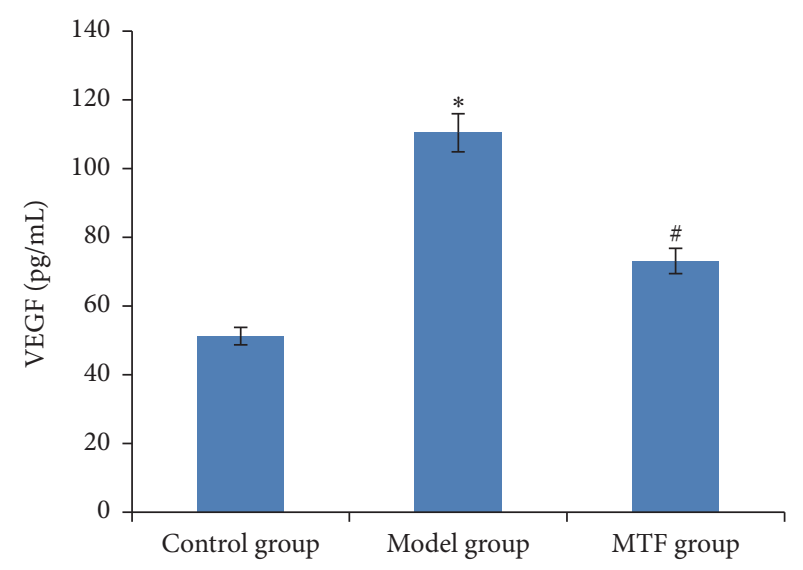

(e)

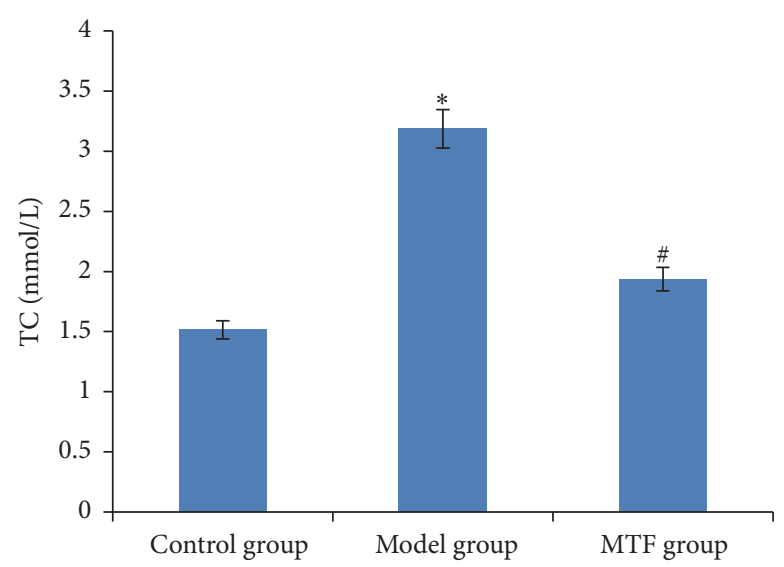

(b)

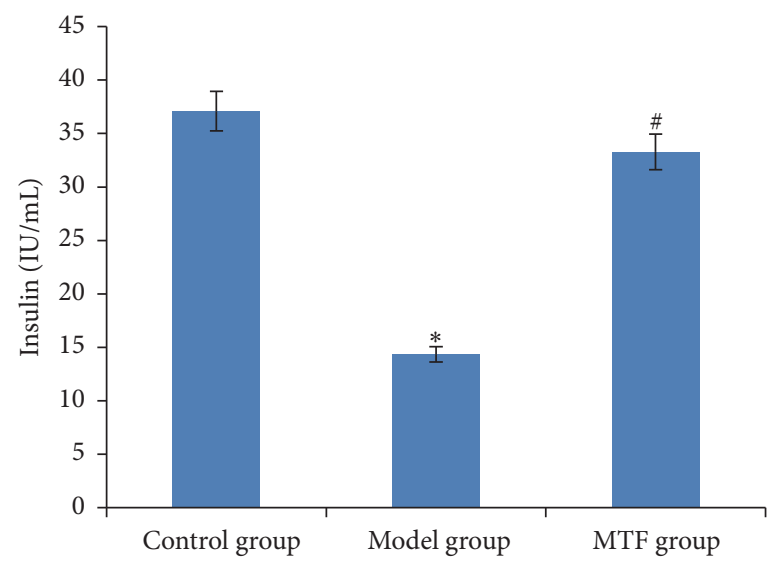

(d)

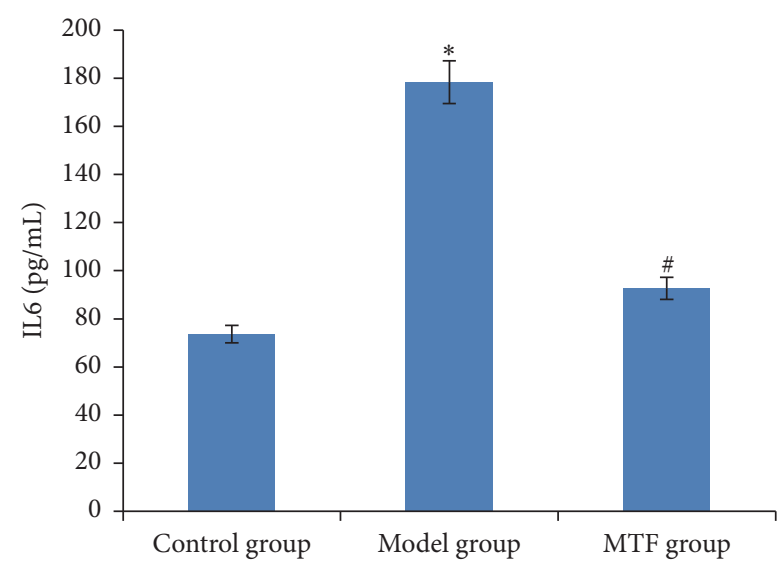

(f)

Figure 1: Blood indicators across three groups. (a) Level of FBG in blood; (b) level of TC in blood; (c) level of TG in blood; (d) level of insulin in blood; (e) level of VEGF in blood; (f) level of IL6 in blood. ${ }^{*}$ Significant difference between the control and model group $(P<0.01)$. ${ }^{\#}$ Significant difference between the model and MTF group $(P<0.01)$.

and, compared to the model group, serum VEGF and IL6 levels were significantly reduced $(P<0.01)$.

Histological images from the model and MTF groups are shown in Figures 2(a), 2(b), 2(c), and 2(d). In the model group, there was a large amount of vascular endothelial cell shedding, and the area of exfoliated endothelial cells was greater than $80 \%$; however, in the MTF group, the exfoliated endothelial cells were distributed locally and the area was lower than $30 \%$. The internal elastic lamina and vascular adventitia did show distinct changes in either group. The 


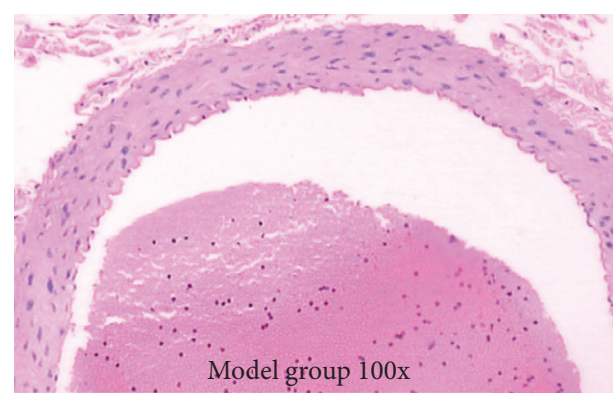

(a)

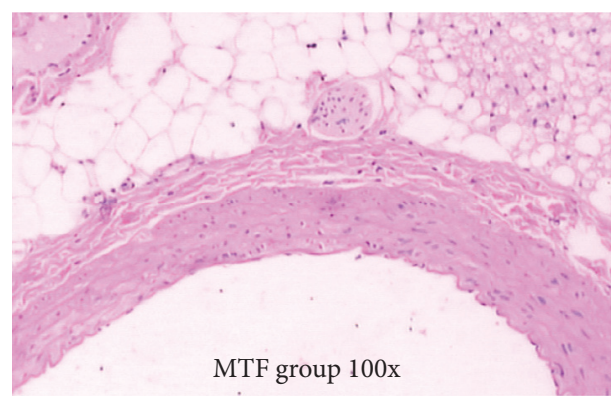

(c)

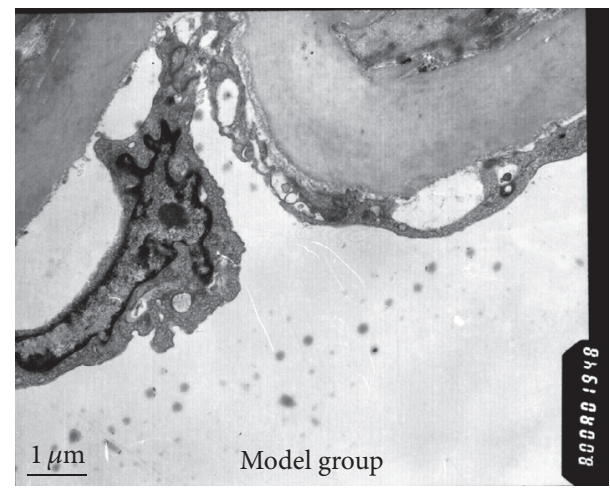

(e)

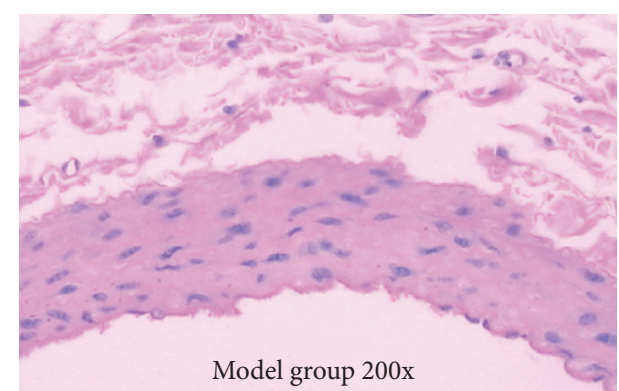

(b)

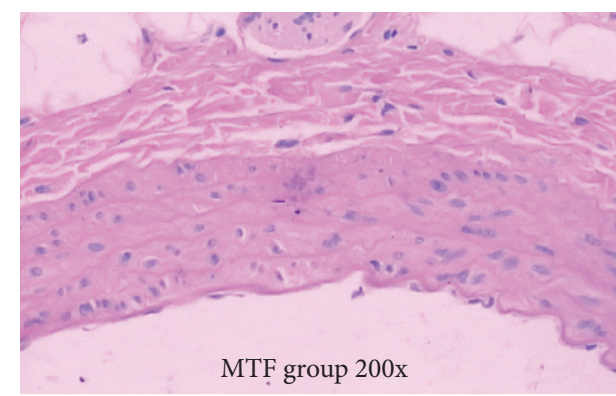

(d)

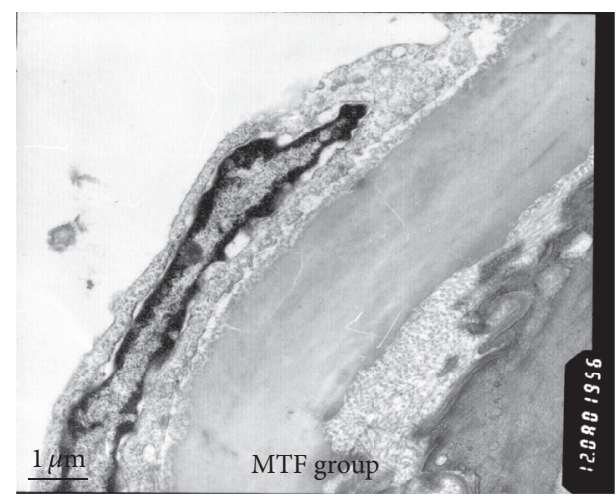

(f)

FiguRE 2: Histomorphological and ultrastructural observations of the femoral artery. (a) Histomorphological observation of model group (magnification $\times 100$ ); (b) histomorphological observation of model group (magnification $\times 200$ ); (c) histomorphological observation of MTF group (magnification $\times 100)$; (d) histomorphological observation of MTF group (magnification $\times 200)$; (e) ultrastructural observation of model group; (f) ultrastructural observation of MTF group.

results of ultrastructural observation are shown in Figures 2(e) and 2(f). In the model group, endothelial cell shedding, nuclear atypia, and chromatin edge accumulation were seen. Compared with the model group, the ultrastructure of endothelial cells in the treatment group was more complete.

3.2. Proteomics Analysis of the Three Groups. To explore the molecular mechanism of MTF, the femoral arteries of the three groups were collected for proteomics analysis using an iTRAQ approach. A total of 764 proteins were identified (see Table S1 in Supplementary Material available online at http://dx.doi.org/10.1155/2016/8076796); there were 78 DPs between the control and model groups and 76 DPs between the model and MTF groups (see Tables S2 and S3). As shown in Figure 3(a), 40 overlapping DPs were found between the two DP comparisons (control versus model and model versus MTF). Deep analysis of these 40 overlapping DPs revealed that 25 DPs were abnormally expressed in the model group and normalized in the MTF group (named MTFnormalized DPs); 11 DPs were upregulated in the model group and downregulated in the MTF group; and 14 DPs were downregulated in the model group and upregulated in the MTF group (Table 1). We further investigated the related biological functions of the MTF-normalized DPs by using the David database. As shown in Table 2, the 25 DPs were significantly enriched in metabolic processes, cellular chemical homeostasis, muscle contraction, the cGMP-PKG signaling pathway, and endocrine and other factor-regulated calcium reabsorption pathways. 
TABLE 1: DPs normalized by MTF treatment.

\begin{tabular}{|c|c|c|c|c|c|}
\hline$\#$ & Accession number & Symbol & Molecular weight & Ratio (model/control) & Ratio (MTF/model) \\
\hline \multirow{11}{*}{$\begin{array}{l}\text { Up expressed in } \\
\text { model then down } \\
\text { expressed in MTF }\end{array}$} & IPI00189809 & Myh6 & $224 \mathrm{kDa}$ & 1.32 & 0.57 \\
\hline & IPI00200352 & Crip2 & $23 \mathrm{kDa}$ & 1.23 & 0.76 \\
\hline & IPI00869592 & Mylk & $217 \mathrm{kDa}$ & 1.23 & 0.81 \\
\hline & IPI00208061 & Atplb3 & $32 \mathrm{kDa}$ & 1.41 & 0.66 \\
\hline & IPI00199872 & Gnas & $46 \mathrm{kDa}$ & 1.41 & 0.71 \\
\hline & IPI00230787 & Car2 & $29 \mathrm{kDa}$ & 1.23 & 0.81 \\
\hline & IPI00231662 & Cyb5r3 & $34 \mathrm{kDa}$ & 1.62 & 0.54 \\
\hline & IPI00231968 & Anxa4 & $36 \mathrm{kDa}$ & 1.23 & 0.76 \\
\hline & IPI00764167 & myh11 & $228 \mathrm{kDa}$ & 1.32 & 0.81 \\
\hline & IPI00390595 & Stk25 & 48 kDa & 1.41 & 0.76 \\
\hline & IPI00421517 & Des & $53 \mathrm{kDa}$ & 1.32 & 0.81 \\
\hline \multirow{14}{*}{$\begin{array}{l}\text { Down expressed in } \\
\text { model then up } \\
\text { expressed in MTF }\end{array}$} & IPI00191090 & Bgn & $42 \mathrm{kDa}$ & 0.76 & 1.32 \\
\hline & IPI00200661 & Fasn & $273 \mathrm{kDa}$ & 0.62 & 1.52 \\
\hline & IPI00205332 & Etfa & $35 \mathrm{kDa}$ & 0.81 & 1.23 \\
\hline & IPI00213036 & $\mathrm{C} 4 \mathrm{a}$ & $192 \mathrm{kDa}$ & 0.71 & 1.23 \\
\hline & IPI00231139 & Tkt & $71 \mathrm{kDa}$ & 0.41 & 2.46 \\
\hline & IPI00231368 & Txn1 & $12 \mathrm{kDa}$ & 0.81 & 1.23 \\
\hline & IPI00326305 & Atplal & $113 \mathrm{kDa}$ & 0.44 & 1.52 \\
\hline & IPI00365985 & Hsp90b1 & $93 \mathrm{kDa}$ & 0.71 & 1.32 \\
\hline & IPI00421539 & Aco2 & $85 \mathrm{kDa}$ & 0.47 & 1.41 \\
\hline & IPI00470254 & Ezr & $69 \mathrm{kDa}$ & 0.81 & 1.23 \\
\hline & IPI00470288 & $\mathrm{Ckb}$ & $43 \mathrm{kDa}$ & 0.71 & 1.32 \\
\hline & IPI00480639 & C3 & $186 \mathrm{kDa}$ & 0.81 & 1.23 \\
\hline & IPI00768626 & Cdh5 & $87 \mathrm{kDa}$ & 0.71 & 1.52 \\
\hline & IPI00196684 & Prkar2a & $46 \mathrm{kDa}$ & 0.57 & 1.32 \\
\hline
\end{tabular}

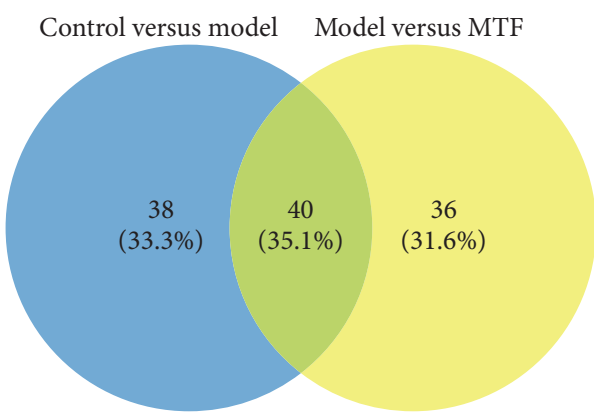

(a)

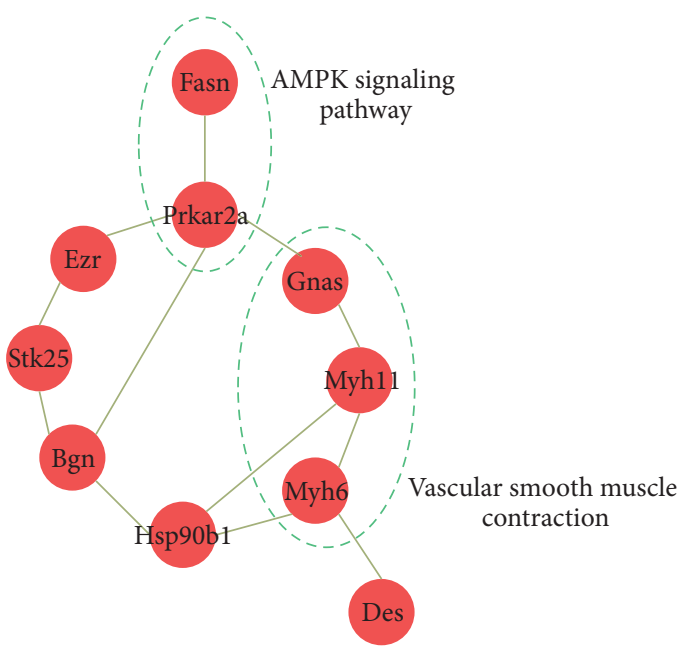

(b)

FIGURE 3: Venny plot of DPs between each group and the PPI network of the DPs normalized by MTF treatment. (a) Venny plot of DPs between each group (control versus model and model versus MTF); (b) PPI network of the DPs normalized by MTF treatment. Line: interaction; red node: DPs. 
TABLE 2: GO and pathway enrichment analysis of the DPs normalized by MTF treatment database.

\begin{tabular}{|c|c|c|c|}
\hline Database & Description & $\begin{array}{l}\text { Protein } \\
\text { number }\end{array}$ & $P$ \\
\hline \multirow{16}{*}{$\begin{array}{l}\text { Gene } \\
\text { ontology }\end{array}$} & Metabolic process & 16 & $5.59 E-04$ \\
\hline & $\begin{array}{l}\text { Single-organism metabolic } \\
\text { process }\end{array}$ & 11 & $2.76 E-03$ \\
\hline & $\begin{array}{l}\text { Small molecule metabolic } \\
\text { process }\end{array}$ & 8 & $4.84 E-03$ \\
\hline & $\begin{array}{l}\text { Regulation of sodium ion } \\
\text { transport }\end{array}$ & 3 & $2.09 E-02$ \\
\hline & $\begin{array}{l}\text { Cellular monovalent } \\
\text { inorganic cation } \\
\text { homeostasis }\end{array}$ & 3 & $2.09 E-02$ \\
\hline & $\begin{array}{l}\text { Cellular potassium ion } \\
\text { homeostasis }\end{array}$ & 2 & $2.09 E-02$ \\
\hline & $\begin{array}{l}\text { Sodium ion export from } \\
\text { cell }\end{array}$ & 2 & $2.09 E-02$ \\
\hline & Chemical homeostasis & 6 & $2.09 E-02$ \\
\hline & $\begin{array}{l}\text { Cellular chemical } \\
\text { homeostasis }\end{array}$ & 5 & $2.09 E-02$ \\
\hline & Membrane repolarization & 2 & $2.09 E-02$ \\
\hline & Inorganic ion homeostasis & 5 & $2.09 E-02$ \\
\hline & $\begin{array}{l}\text { Positive regulation of } \\
\text { striated muscle contraction }\end{array}$ & 2 & $2.50 E-02$ \\
\hline & $\begin{array}{l}\text { Regulation of biological } \\
\text { quality }\end{array}$ & 8 & $2.50 E-02$ \\
\hline & $\begin{array}{l}\text { Cellular sodium ion } \\
\text { homeostasis }\end{array}$ & 2 & $3.38 E-02$ \\
\hline & $\begin{array}{l}\text { Response to organic } \\
\text { substance }\end{array}$ & 8 & $3.38 E-02$ \\
\hline & Muscle contraction & 3 & $4.78 E-02$ \\
\hline \multirow{13}{*}{$\begin{array}{l}\text { KEGG } \\
\text { pathway }\end{array}$} & Gastric acid secretion & 5 & $2.44 E-06$ \\
\hline & $\begin{array}{l}\text { Proximal tubule } \\
\text { bicarbonate reclamation }\end{array}$ & 3 & $2.47 E-04$ \\
\hline & Thyroid hormone synthesis & 3 & $4.23 E-03$ \\
\hline & Bile secretion & 3 & $4.23 E-03$ \\
\hline & Cardiac muscle contraction & 3 & $4.50 E-03$ \\
\hline & Pancreatic secretion & 3 & $6.79 E-03$ \\
\hline & $\begin{array}{l}\text { Thyroid hormone signaling } \\
\text { pathway }\end{array}$ & 3 & $1.03 E-02$ \\
\hline & $\begin{array}{l}\text { Adrenergic signaling in } \\
\text { cardiomyocytes }\end{array}$ & 3 & $1.61 E-02$ \\
\hline & $\begin{array}{l}\text { cGMP-PKG signaling } \\
\text { pathway }\end{array}$ & 3 & $2.20 E-02$ \\
\hline & $\begin{array}{l}\text { Aldosterone-regulated } \\
\text { sodium reabsorption }\end{array}$ & 2 & $2.24 E-02$ \\
\hline & $\begin{array}{l}\text { Carbohydrate digestion and } \\
\text { absorption }\end{array}$ & 2 & $2.24 E-02$ \\
\hline & $\begin{array}{l}\text { Endocrine and other } \\
\text { factor-regulated calcium } \\
\text { reabsorption }\end{array}$ & 2 & $2.50 E-02$ \\
\hline & Mineral absorption & 2 & $2.50 E-02$ \\
\hline
\end{tabular}

3.3. PPI Network of the DPs. To investigate the relationship between the 25 MTF-normalized DPs, we constructed a PPI network using a 10.0 database. As shown in Figure 3(b), the PPI network included 10 DPs and 12 interactions between them. In this PPI network, Fasn and Prkar2a and their interaction were found to be involved in the AMPK signaling

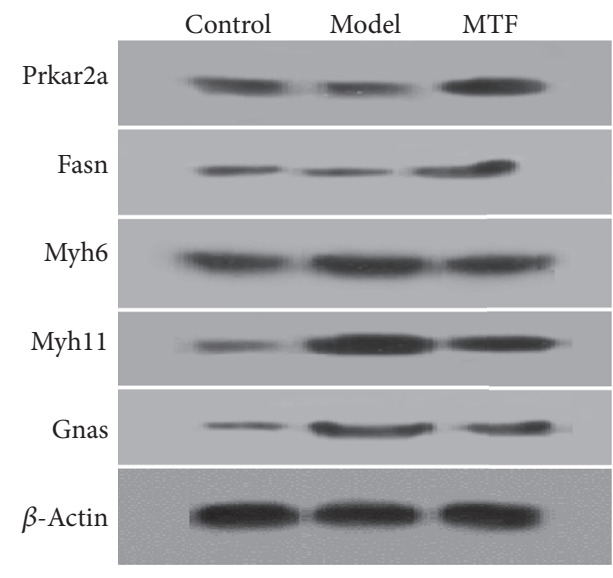

FIGURE 4: Western blots of the five DPs (Prkar2a, Fasn, Gnas, Myh11, and Myh6).

pathway; these two DPs were downregulated in the model group and upregulated in the MTF group. Gnas, Myh11, and Myh6 and their interactions were also found to be involved in vascular smooth muscle contraction; these three DPs were upregulated in the model group and downregulated in the MTF group.

3.4. Validation of Representative DPs by Western Blotting. To verify proteomic analysis data, five DPs (Fasn, Prkar2a, Gnas, Myhll, and Myh6) were validated using Western blotting. As shown in Figure 4, the expression of all five DPs was consistent with the iTRAQ data. Fasn and Prkar2a were downregulated in the model group and upregulated in the MTF group. Gnas, Myh11, and Myh6 were upregulated in the model group and downregulated in the MTF group.

\section{Discussion}

Lower limb macroangiopathy is a major complication associated with diabetes mellitus and has been shown to precede amputation in up to $90 \%$ of cases [16]. Current studies have indicated that continuous metabolic and chronic hemodynamic alterations in the blood of patients with T2DM could damage the endothelium, resulting in structural and functional changes and leading to thickening of the basement membrane and sclerosis of vascular walls, which can cause lower limb macroangiopathy $[17,18]$. Many biological processes are involved in the pathogenesis of macroangiopathy, including oxidative stress, collagen deposition, angiogenesis, ECM remodeling, and vascular remodeling [19-21]. Improvement of these biological processes may be fundamental to treating lower limb macroangiopathy in T2DM.

In this study, we evaluated the efficacy and the molecular mechanism underlying MTF treatment. Our results showed that MTF treatment significantly reduced serum FBG, TG, and TC levels and significantly increased serum insulin levels in GK rats. We also found that MTF treatment significantly reduced levels of VEGF, which is associated with atherosclerosis, vascular ischemia, hypoxia, and AGE accumulation in 
T2DM [22]. This reduction in VEGF indicates that MTF treatment may address antiatherosclerosis. Moreover, we found that MTF treatment also significantly reduced IL6 levels in GK rats, suggesting anti-inflammation effects [23], since IL6 is associated with vascular smooth muscle contraction in rat models of diabetes [24]. Based on visual inspections, we found a significant reduction in vascular endothelial cell shedding and improvement in endothelium injuries. Ultrastructural observation showed that MTF treatment also significantly improved necrosis in vascular endothelial cells. Taken together, these results indicate that MTF alleviates angiogenesis and affects vascular remodeling.

To characterize the molecular mechanism of MTF treatment, a proteomics analysis was used to explore multitarget characteristics. Abnormal expression of 25 DPs was normalized by MTF treatment in the GK rats. Functional enrichment analysis showed that these DPs were significantly involved in cellular chemical homeostasis, muscle contraction, cGMPPKG signaling pathways, and endocrine and other factorregulated calcium reabsorption, all of which are significantly associated with T2DM [25-31]. Moreover, we constructed a PPI network of these DPs, and, in this PPI network, Fasn and Prkar2a and their interaction were found to be involved in the AMPK signaling pathway. These 2 proteins have been proven to play key roles in macroangiopathy in T2DM. Fasn is a fatty-acid synthase, which may target endothelial nitricoxide synthase (eNOS) in the plasma membrane by adding palmitate to eNOS; in this scenario, eNOS could generate $\mathrm{NO}$ in vascular tissue, and, in its absence, $\mathrm{NO}$ could decrease vasodilatation effectively leading to limb/nerve ischemia and macroangiopathy in T2DM [29]. Prkar2a is known to be a key upstream regulatory factor for Fasn $[30,32]$. Three proteins (Gnas, Myhll, and Myh6) were found to be involved in vascular smooth muscle contraction, which is associated with vascular ischemia and poor local blood flow in macroangiopathy in T2DM [24]. These results indicate that MTF treatment could significantly improve vascular smooth muscle contraction.

T2DM is a complex disease that involves protein imbalance and the disturbance of multiple biological pathways. This study has shown that MTF can normalize many proteins and corresponding biological processes/pathways associated with T2DM. In accordance with our experimental results, we speculate a mechanistic pathway in vascular tissue that could be affected by MTF treatment (Figure 5). In this pathway, MTF could upregulate the expression of Prkar2a and Fasn, leading to eNOS palmitoylation, which would increase NO concentration and finally improve macroangiopathy. In addition, MTF could downregulate the expression of Gnas, Myhl1, and Myh6 (which are associated with vascular ischemia), thus leading to improvement in macroangiopathy.

\section{Competing Interests}

The authors declare that they have no competing interests.

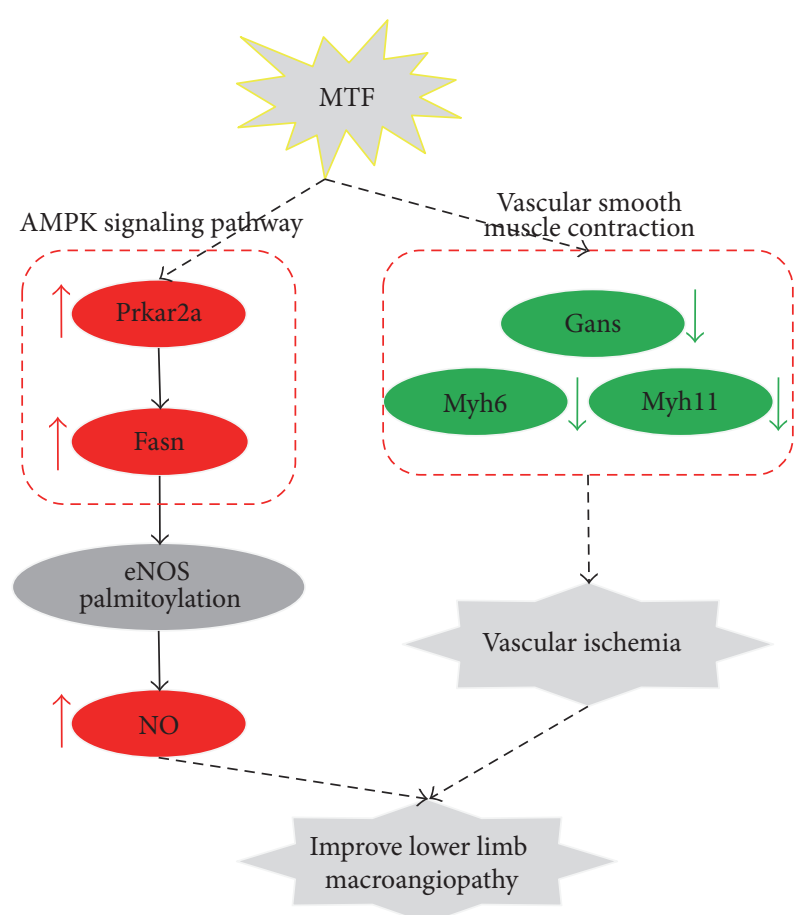

FIGURE 5: Mechanism of MTF treatment for lower limb macroangiopathy in T2DM.

\section{References}

[1] International Diabetes Federation (IDF), IDF Diabetes Atlas, Update 2015, International Diabetes Federation (IDF), Brussels, Belgium, 7th edition, 2015.

[2] K. Markakis, F. L. Bowling, and A. J. M. Boulton, "The diabetic foot in 2015: an overview," Diabetes/Metabolism Research and Reviews, vol. 32, Supplement 1, pp. 169-178, 2016.

[3] W. Clayton Jr. and T. A. Elasy, "A review of the pathophysiology, classification, and treatment of foot ulcers in diabetic patients," Clinical Diabetes, vol. 27, no. 2, pp. 52-58, 2009.

[4] R. Sinha, W. J. A. van den Heuvel, and P. Arokiasamy, "Factors affecting quality of life in lower limb amputees," Prosthetics and Orthotics International, vol. 35, no. 1, pp. 90-96, 2011.

[5] S. Noor, R. U. Khan, and J. Ahmad, "Understanding diabetic foot infection and its management," Diabetes \& Metabolic Syndrome: Clinical Research \& Reviews, 2016.

[6] A. L. Lee, B. C. Chen, C. H. Mou, M. F. Sun, and H. R. Yen, "Association of Traditional Chinese Medicine therapy and the risk of vascular complications in patients with type II diabetes mellitus: a nationwide, retrospective, Taiwaneseregistry, cohort study," Medicine, vol. 95, no. 3, article e2536, 2016.

[7] G.-D. Sun, C.-Y. Li, W.-P. Cui et al., "Review of herbal Traditional Chinese Medicine for the treatment of diabetic nephropathy," Journal of Diabetes Research, vol. 2016, Article ID 5749857, 18 pages, 2016.

[8] J. C. W. Tam, C. H. Ko, C. Zhang et al., "Comprehensive proteomic analysis of a Chinese 2-herb formula (Astragali Radix and Rehmanniae Radix) on mature endothelial cells," Proteomics, vol. 14, no. 17-18, pp. 2089-2103, 2014.

[9] S.-S. Luan, F. Yu, B.-Y. Li et al., "Quantitative proteomics study of protective effects of grape seed procyanidin B2 on diabetic 
cardiomyopathy in $\mathrm{db} / \mathrm{db}$ mice," Bioscience, Biotechnology and Biochemistry, vol. 78, no. 9, pp. 1577-1583, 2014.

[10] Q. Cai, B. Li, F. Yu et al., "Investigation of the protective effects of phlorizin on diabetic cardiomyopathy in $\mathrm{db} / \mathrm{db}$ mice by quantitative proteomics," Journal of Diabetes Research, vol. 2013, Article ID 263845, 9 pages, 2013.

[11] F. Yu, B.-Y. Li, X.-L. Li et al., "Proteomic analysis of aorta and protective effects of grape seed procyanidin $\mathrm{B} 2 \mathrm{in} \mathrm{db} / \mathrm{db}$ mice reveal a critical role of milk fat globule epidermal growth factor8 in diabetic arterial damage," PLoS ONE, vol. 7, no. 12, Article ID e52541, 2012.

[12] S. Cai, W. Sun, Y. Fan et al., "Effect of mulberry leaf (Folium Mori) on insulin resistance via IRS-1/PI3K/Glut-4 signalling pathway in type 2 diabetes mellitus rats," Pharmaceutical Biology, vol. 54, no. 11, pp. 2685-2691, 2016.

[13] L. Ma, L. Huang, H. Pei et al., "Pharmacological effects of the water fraction of key components in the traditional chinese prescription Mai Tong Fang on 3T3-L1 adipocytes and ob/ob Diabetic Mice," Molecules, vol. 19, no. 9, pp. 14687-14698, 2014.

[14] D. W. Huang, B. T. Sherman, and R. A. Lempicki, "Systematic and integrative analysis of large gene lists using DAVID bioinformatics resources," Nature Protocols, vol. 4, no. 1, pp. 44-57, 2009.

[15] D. Szklarczyk, A. Franceschini, S. Wyder et al., "STRING v10: protein-protein interaction networks, integrated over the tree of life," Nucleic Acids Research, vol. 43, no. 1, pp. D447-D452, 2015.

[16] S. Jhamb, V. N. Vangaveti, and U. H. Malabu, "Genetic and molecular basis of diabetic foot ulcers: clinical review," Journal of Tissue Viability, vol. 25, no. 4, pp. 229-236, 2016.

[17] E. M. Yubero-Serrano, J. Delgado-Lista, J. F. Alcala-Diaz et al., "A dysregulation of glucose metabolism control is associated with carotid atherosclerosis in patients with coronary heart disease (CORDIOPREV-DIAB study)," Atherosclerosis, vol. 253, pp. 178-185, 2016.

[18] F. Semeraro, A. Cancarini, R. dell'Omo, S. Rezzola, M. R. Romano, and C. Costagliola, "Diabetic retinopathy: vascular and inflammatory disease," Journal of Diabetes Research, vol. 2015, Article ID 582060, 16 pages, 2015.

[19] P. Madeddu, "Therapeutic angiogenesis and vasculogenesis for tissue regeneration," Experimental Physiology, vol. 90, no. 3, pp. 315-326, 2005.

[20] U. Förstermann and T. Münzel, "Endothelial nitric oxide synthase in vascular disease: from marvel to menace," Circulation, vol. 113, no. 13, pp. 1708-1714, 2006.

[21] R. Blakytny and E. Jude, "The molecular biology of chronic wounds and delayed healing in diabetes," Diabetic Medicine, vol. 23, no. 6, pp. 594-608, 2006.

[22] R. L. Avery and G. M. Gordon, "Systemic safety of prolonged monthly anti-vascular endothelial growth factor therapy for diabetic macular edema: a systematic review and metaanalysis," JAMA Ophthalmology, vol. 134, no. 1, pp. 21-29, 2016.

[23] D. Baltzis, I. Eleftheriadou, and A. Veves, "Pathogenesis and treatment of impaired wound healing in diabetes mellitus: new insights," Advances in Therapy, vol. 31, no. 8, pp. 817-836, 2014.

[24] W.-B. Tang, Y.-Q. Zhou, T. Zhao et al., "Effect of interleukin-6 (IL-6) on the vascular smooth muscle contraction in abdominal aorta of rats with streptozotocin-induced diabetes," Chinese Journal of Physiology, vol. 54, no. 5, pp. 318-323, 2011.

[25] W. J. Jeffcoate and K. G. Harding, "Diabetic foot ulcers," The Lancet, vol. 361, no. 9368, pp. 1545-1551, 2003.
[26] L. B. Bockus and K. M. Humphries, "CAMP-dependent protein kinase (PKA) signaling is impaired in the diabetic heart," Journal of Biological Chemistry, vol. 290, no. 49, pp. 2925029258, 2015.

[27] S. Guner, E. Arioglu, A. Tay et al., "Diabetes decreases mRNA levels of calcium-release channels in human atrial appendage," Molecular and Cellular Biochemistry, vol. 263, no. 1, pp. 143-150, 2004.

[28] S. Zhu, R. S. Guleria, C. M. Thomas et al., "Loss of myocardial retinoic acid receptor $\alpha$ induces diastolic dysfunction by promoting intracellular oxidative stress and calcium mishandling in adult mice," Journal of Molecular and Cellular Cardiology, vol. 99, pp. 100-112, 2016.

[29] X. Wei, J. G. Schneider, S. M. Shenouda et al., "De Novo lipogenesis maintains vascular homeostasis through endothelial nitricoxide synthase (eNOS) palmitoylation," Journal of Biological Chemistry, vol. 286, no. 4, pp. 2933-2945, 2011.

[30] J. Chen, J. Ren, Q. Jing et al., “TSH/TSHR signaling suppresses Fatty Acid Synthase (FASN) expression in adipocytes," Journal of Cellular Physiology, vol. 230, no. 9, pp. 2233-2239, 2015.

[31] C. R. Triggle, H. Ding, T. J. Anderson, and M. Pannirselvam, "The endothelium in health and disease: a discussion of the contribution of non-nitric oxide endothelium-derived vasoactive mediators to vascular homeostasis in normal vessels and in type II diabetes," Molecular and Cellular Biochemistry, vol. 263, no. 1, pp. 21-27, 2004.

[32] T. A. M. Almabrouk, M. A. Ewart, I. P. Salt, and S. Kennedy, "Perivascular fat, AMP-activated protein kinase and vascular diseases," British Journal of Pharmacology, vol. 171, no. 3, pp. 595-617, 2014. 


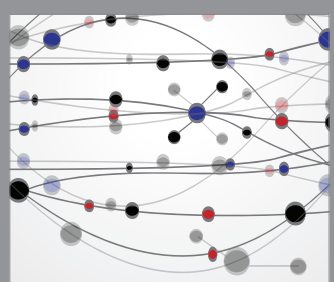

The Scientific World Journal
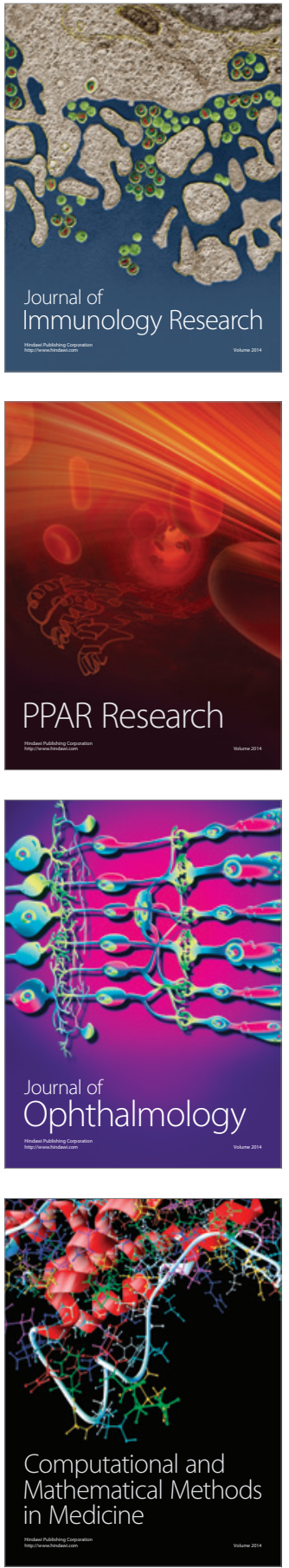

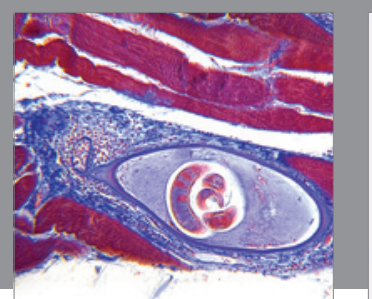

Gastroenterology Research and Practice

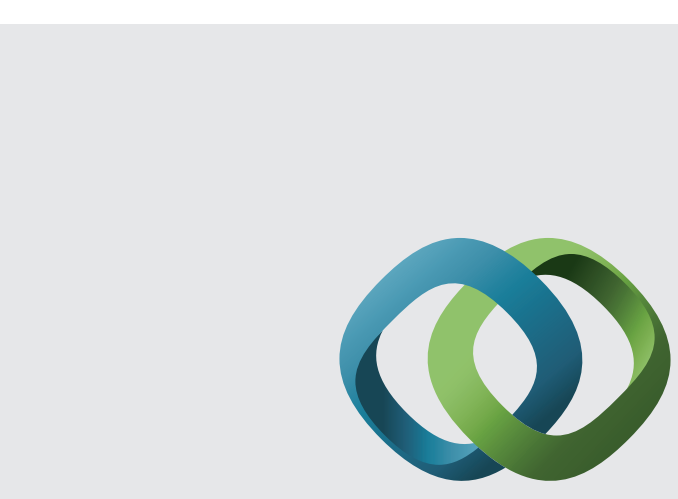

\section{Hindawi}

Submit your manuscripts at

http://www.hindawi.com
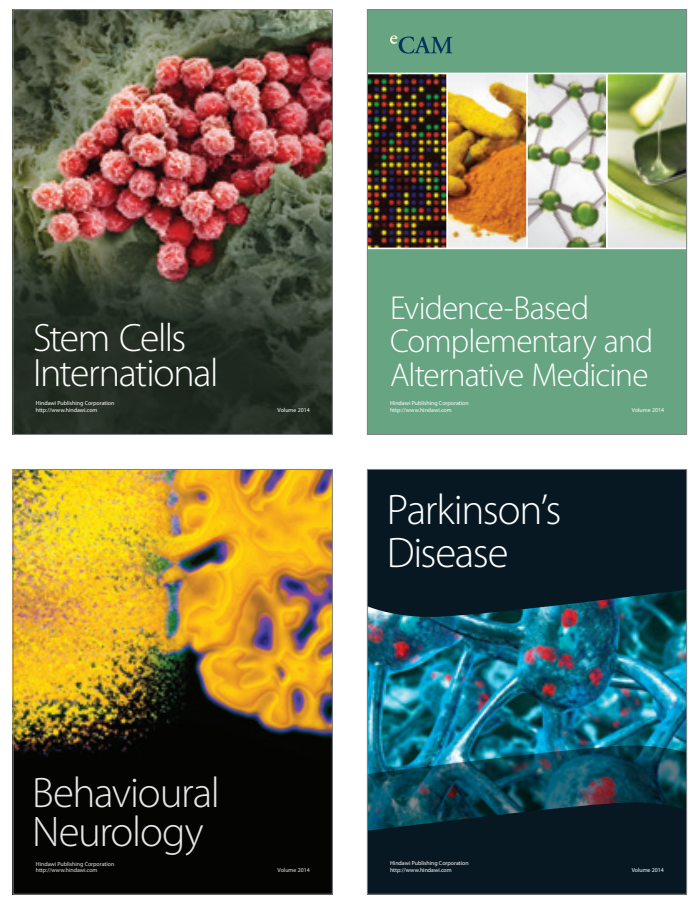
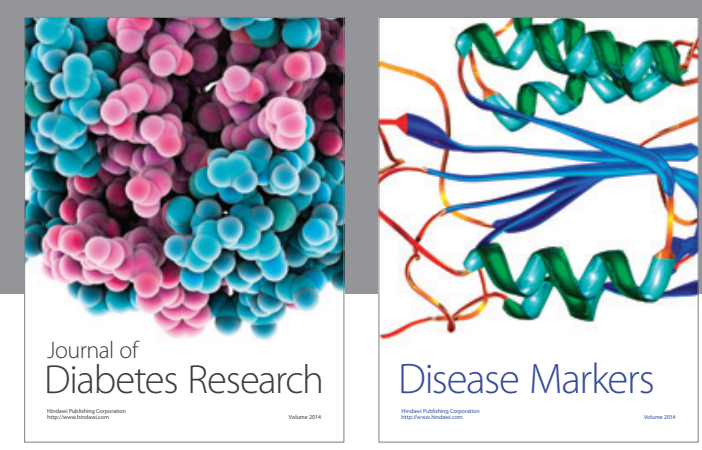

Disease Markers
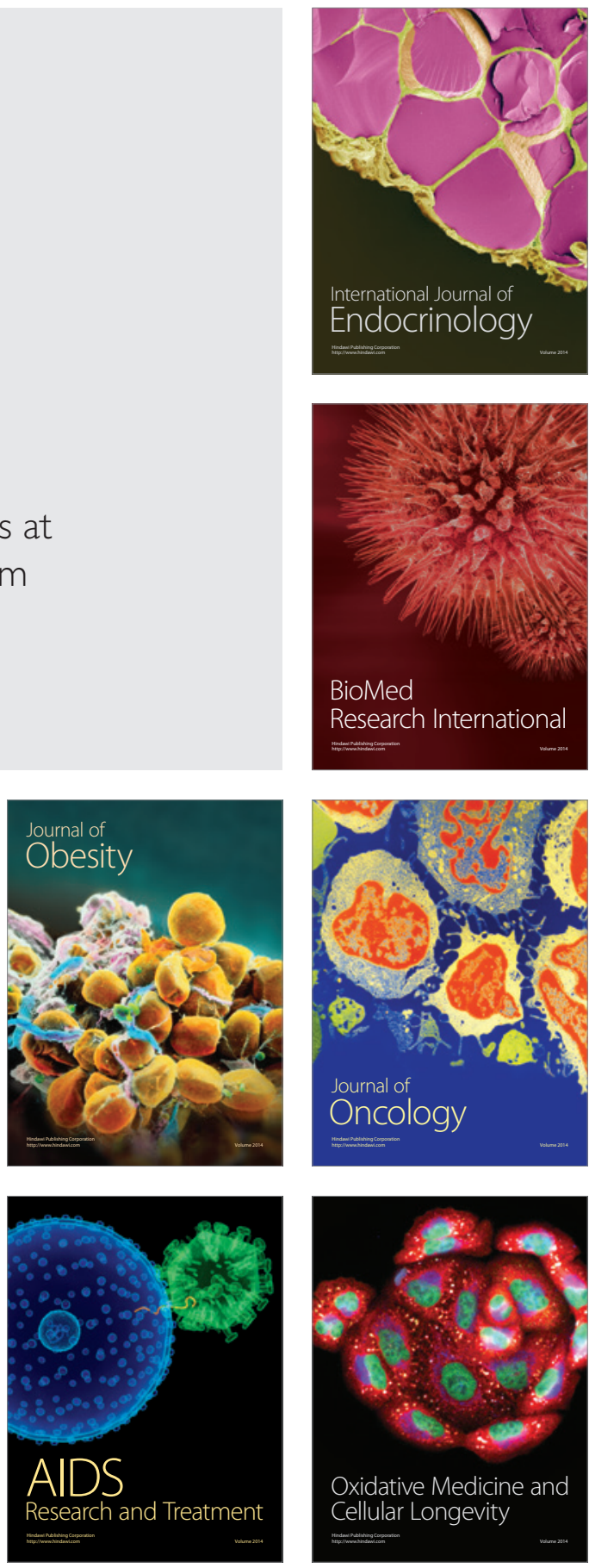